\title{
Prognostic effect of factors involved in revised Tokuhashi score system for patients with spinal metastases: a systematic review and Meta-analysis
}

Xiong-gang Yang ${ }^{1}$, Deng-xing Lun², Yong-cheng Hu ${ }^{1 *}$ (D), Yong-heng $\mathrm{Liu}^{3}$, Feng Wang ${ }^{3}$, Jiang-tao Feng ${ }^{3}$, Kun-Chi Hua ${ }^{3}$, Li Yang ${ }^{3}$, Hao Zhang ${ }^{3}$, Ming-you Xu ${ }^{3}$ and Hao-ran Zhang ${ }^{3}$

\begin{abstract}
Background: Cancer patients' survival time has obviously improved, with the development of systemic treatment techniques. However, the probability of metastases to the vertebrae has also been increased which makes some adverse effects on patients' quality of life. The prediction of survival plays a key role in choosing therapeutic modality, and Tokuhashi Score was established as one of the most commonly used predictive systems for spinal metastases. Thus, this study was conducted to identify the prognostic effect of factors involved in revised Tokuhashi Score (RTS).

Methods: Two investigators independently retrieved relevant literature on platforms of PubMed, Embase and Cochrane Library. We identified eligible studies through title/abstract and full-text perusing. Data was extracted including general information of studies, participants' characteristics, therapeutic modality, overall survival and prognostic effect of factors. Hazard ratio (HR) for each factor was synthesized if available through fixed- or randomeffect models as appropriate.

Results: A total of 63 eligible studies with 10,411 participants were identified. Overall, cases with thyroid cancer had the highest survival rate, while the ones with non-small cell lung cancer and hepatocellular carcinoma lived for the shorted survival time. Performance status, bone metastasis, number of involved vertebrae, visceral metastasis, primary tumor and neurological status were regarded as significant predictors in $71.4,40.0,18.2,63.4,73.1$ and $44.7 \%$ of the involved studies respectively. Thirty-eight articles were included in meta-analysis, and prognostic effects of five factors (apart from primary tumor) were analyzed. Factors were all proved to be significant except comparisons between KPS (Karnofsky Performance Status) 10-40 VS. 50-70 and single VS. multiple spinal metastases.

Conclusion: All factors of RTS were significant on prognosis predicting and should be considered when choosing therapeutic modality for spinal metastases. What's more, we believe that more accurate prognosis may be obtained after removal of the cut-offs for KPS 10-40 VS. 50-70 and single VS. multiple involved vertebrae.
\end{abstract}

Keywords: Spinal metastasis, Prognostic factor, Overall survival, Revised Tokuhashi score

\footnotetext{
* Correspondence: yongchenghu@126.com

'Department of Bone Tumor of Tianjin Hospital, Tianjin 300211, China

Full list of author information is available at the end of the article
}

(c) The Author(s). 2018 Open Access This article is distributed under the terms of the Creative Commons Attribution 4.0 International License (http://creativecommons.org/licenses/by/4.0/), which permits unrestricted use, distribution, and reproduction in any medium, provided you give appropriate credit to the original author(s) and the source, provide a link to the Creative Commons license, and indicate if changes were made. The Creative Commons Public Domain Dedication waiver (http://creativecommons.org/publicdomain/zero/1.0/) applies to the data made available in this article, unless otherwise stated. 


\section{Background}

With the improvements of systemic treatment techniques, cancer patients' survival has obviously extended. However, the probability of metastases to the vertebrae has greatly increased, up to about 70\%, which would make adverse effects on patients' life quality $[1,2]$. Patients suffered from spinal metastases usually have symptoms of intractable pain, neurological deficit and spinal instability, as the results of metastatic spinal cord compression (MSCC). In general, most of these patients are likely to benefit from aggressive surgery interventions while some are not if their life expectancies are extremely limited. Hence, for selecting of the optimal therapeutic modality, prognostic factors of the overall survival should be identified and taken into consideration.

Many studies have attempted to identify prognostic factors that predict survival of patients with spinal metastasis, and some handy scores have been established such as Tokuhashi [3, 4], Sioutos [5] and Tomita [6], Bauer [7], North [8] and Van der Linden [9]. Tokuhashi score is one of the most popularly used score systems for spinal metastases and most commonly reported in literature, which was originally established in 1990 and finally revised in 2005 [3, 4]. This score includes the following prognostic factors: performance status, bone metastases, number of involved vertebrae, visceral metastases, primary tumor type and neurological status. The type of primary tumor was scored between 0 and 5 , while the other factors were scored between 0 and 2, which was added up to a maximum score of 15 (Table 1). According to this scoring system, if the total score is ranged $0-8$, the predicted survival time will be less than 6 months and the conservative treatment or palliative surgery will be the optimal therapeutic modalities. For patients with a score of $12-15$, the predicted survival time will be more than 12 months and more aggressive excisional surgery should be selected. And for patients with a score of 9-11, the predicted survival will be 6-12 months and palliative surgery or excisional surgery (a single vertebra was involved with no metastasis to major internal organs) will be recommended. The original authors have performed a validation study on the revised Tokuhashi Score (RTS) and shown an excellent accuracy as high as $87.9 \%$ between the predicted and actual survival. However, the accuracy of RTS in predicting the life expectancy for spinal metastases remain unsatisfying. Especially when cancer patients' overall survival has been greatly improved because of more curative therapies (i.e. targeted therapy), the consistence and accuracy of RTS further decreased. As reported by Quraishi et al. [10], the prognostic criteria using RTS could only be moderately useful in predicting actual survival (66\%). Pointillart et al. [11] also concluded from a prospective study that neither the original nor revised Tokuhashi scores were reliable in predicting survival in European population. The predictive value of the RTS was found to
Table 1 Revised Tokuhashi Score System for the Prognosis of Spinal Metastasis

\begin{tabular}{|c|c|}
\hline Factors & Score \\
\hline \multicolumn{2}{|l|}{ General condition (Karnofsky Performance Status, KPS) } \\
\hline Poor (KPS 10-40) & 0 \\
\hline Moderate (KPS 50-70) & 1 \\
\hline Good (KPS 80-100) & 2 \\
\hline \multicolumn{2}{|l|}{ Extraspinal bone metastases } \\
\hline$\geq 3$ & 0 \\
\hline $1-2$ & 1 \\
\hline 0 & 2 \\
\hline \multicolumn{2}{|l|}{ No. of metastases in the vertebral body } \\
\hline$\geq 3$ & 0 \\
\hline 2 & 1 \\
\hline 1 & 2 \\
\hline \multicolumn{2}{|l|}{ Metastases to the major internal organs } \\
\hline Unremovable & 0 \\
\hline Removable & 1 \\
\hline No metastases & 2 \\
\hline \multicolumn{2}{|l|}{ Primary site of the cancer } \\
\hline Lung, osteosarcoma, stomach, bladder, esophagus, pancreas & 0 \\
\hline Liver, gallbladder, unidentified & 1 \\
\hline Others & 2 \\
\hline Kidney, uterus & 3 \\
\hline Rectum & 4 \\
\hline Thyroid, breast, prostate, carcinoid tumor & 5 \\
\hline \multicolumn{2}{|l|}{ Neurological Status } \\
\hline Complete (Frankel A, B) & 0 \\
\hline Incomplete (Frankel C, D) & 1 \\
\hline None (Frankel E) & 2 \\
\hline
\end{tabular}

be less than $60 \%$, and the prognostic effect of the factors showed conflicting results. For example, Tokuhashi [3, 4] included neurological deficit in the score, whereas Tomita [6], Bauer [7], North [8] and Van der Linden [9] did not.

Thus, the current study aimed to assess the effect of different parameters in RTS for predicting survival of patients with spinal metastases, and modify on the contents of RTS according to the significance of each parameter.

\section{Methods}

\section{Data sources and searches}

This review was conducted according to the guidelines outlined in Preferred Reporting Items for Systematic Reviews and Meta-analysis (PRISMA) statement. Two individual researchers (Yang XG and Lun DX) conducted platform searches on the PubMed, Embase and Cochrane Library. Literature retrieving was carried out through a 
combined searching of subject terms ("MeSH" on PubMed and "Emtree" on Embase) and free terms on PubMed and Embase, and through keywords searching on Cochrane Library. Searching strategies used on PubMed and Embase was presented in Additional file 1: Appendix 1. And the searching on Cochrane Library was conducted with the following keywords: "spinal metastasis; overall survival; prognostic factor". Additionally, some else reference studies of relative articles and reviews were screened and hand-searched for possible inclusion.

\section{Inclusion and exclusion criteria for studies}

Complete texts published between January 1997 and October 2017 (over the last two decades) with designs of cohort or case-control study approaching the survival and prognostic effect of factors included in RTS for patients with spinal metastases were included. The publication language was restricted in English but there were no limitations on the participants' nationalities.

Studies would be excluded for the following reasons: (1) literature review, systematic review and/or meta analysis and letter to editors; (2) studies with less than 10 participants; (3) studies using repeated cohorts; (4) studies with high risk of bias according to the quality assessment; (5) duplicated studies.

\section{Study selection}

After all duplicates were recognized and merged together by the software of EndNote X7 version 17.0 (Clarivate Analytics, Philadelphia, USA), the remained titles and abstracts were screened. Then, full texts of potentially relevant papers were obtained and assessed by full-text perusing for eligibility. The whole process of selection was strictly followed with the inclusion and exclusion criteria by two review authors (Yang XG and Lun $D X$ ) independently. Discrepancies in study selection between the two reviewers were handled by face-to-face discussion or judged by the third reviewer (Liu YH).

\section{Data extraction and quality assessment}

Data was extracted by the two review authors pair independently and entered into a pre-built Microsoft Excel spreadsheet. Collected data included the following information: (1) characteristics of studies (title, author, publication year, country, study period, study design and quality of study), (2) participants' characteristics (age, percentage of male, number of patients, number of patients with MSCC, primary tumor and spinal metastasis location); (3) therapeutic modality; (4) follow-up and overall survival; (5) prognostic effect of the factors and effect sizes for hazard ratio (HR) combined with their 95\% confidence interval (95\%CI) representing the prognostic value of factors included in RTS. We figured out causes of diversities on obtained information and resolved disagreements after discussion.
The Newcastle-Ottawa Scale (NOS) [12] was used for the assessment on risk of bias of the studies. This scale employs a 9 stars system that assesses three domains: patient selection, comparability of study groups and ascertainment of study outcome. Studies with a score of 8-9 stars have low risk of bias whereas scores of 6-7 mean medium bias risk and a score of 5 or less than 5 indicates a high chance of bias. Studies with a score of $\leq 5$ stars would be excluded from this study.

\section{Quantitative data analysis}

All recorded HRs and CI95\% from eligible literature was pooled by an exploratory time-to-event meta-analysis with a random- or fixed-effect model as appropriate and heterogeneity was tested with $\mathrm{I}^{2}$ [13]. In case with significant heterogeneity $\left(\mathrm{I}^{2}>50 \%\right)$, random-effect model would be employed, while fixed-effect model would be selected when presenting with excellent homogeneity $\left(\mathrm{I}^{2}<50 \%\right)$. A test for the pooled effect sizes by $\mathrm{Z}$ test was performed and statistical significance was defined at a two-sided $P$ value of less than 0.05 . A sensitivity analysis would be performed when significant heterogeneity existing and studies causing instability would be removed. Publication bias was assessed with Begg's and Egger's regression asymmetry test $(p<$ 0.050 and $p<0.100$ were considered to be with significant publication bias respectively) [14]. In case with significant publication bias, a nonparametric trim and fill method will be performed to rectify the bias [15]. The whole process of meta-analysis was performed by Stata version 13.0 (StataCorp LLC, College Station, Texas, USA).

\section{Results}

\section{Search result and study selection}

The flow chart of eligible literature selection was shown in Fig. 1. The initial searching on electronic platforms yielded a total of 2194 studies and another 3 articles were obtained by hand-searching. After exclusion of 293 duplicates, 1904 articles remained. Then by preliminary glancing over titles and abstracts and further perusing at full-texts, a number of 1503 and 338 articles were excluded respectively. The 338 full texts were excluded with the following reason: 304 studies didn't involve prognostic effect of the factors involved in Tokuhashi Score; 28 studies were literature or systematic reviews; 3 studies of Lei [16-18] used repeated patients cohort, thus only the one [18] identified primary tumor histology as non-small cell lung cancer(NSCLC) was included; and another 4 studies of Rades [19-22] were also excluded for using repeated patients cohorts with other studies. Finally, 63 studies [6, $8,9,18,24-72,74-82]$ with 10,411 participants and 38 studies $[8,9,18,26,28-38,40,41,43,44,46,47,49,51-$ $53,56,58,60,63,64,66,69,71,76,78-81]$ with 7462 participants were included in the qualitative and quantitative synthesis respectively. 


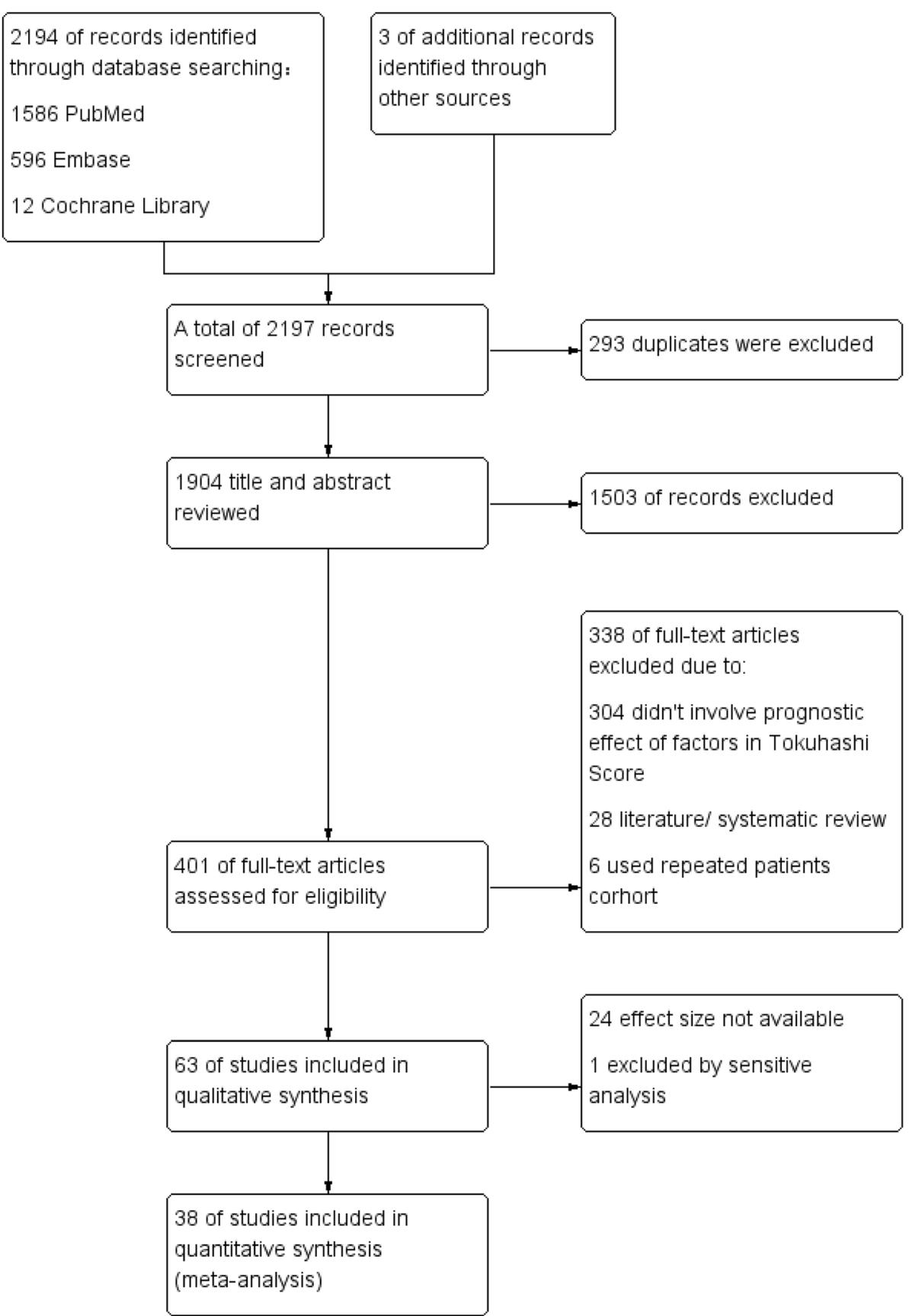

Fig. 1 Flowchart of studies identification and selection

\section{General information of studies}

Summary of individual study was listed in Table 2. Majority of the studies were of favourable quality assessed by NOS, with an average score of $7.8 \pm 1.0$ stars. None of the studies were excluded by quality assessment, which means no studies showed high risk of bias (NOS $\leq 5$ stars). As for the delimitation, 57 and 4 studies were retrospective and prospective cohorts respectively, but only 1 each was case-control study and semi-retrospective cohort with a prospective manner on part of the information collection. Primary tumor histology was various among included studies, with 29 non-specified tumor type (7577 patients), 8 prostate cancer (842 patients), 6 non-small cell lung cancer (NSCLC, 667 patients), 6 breast cancer (648 patients), 4 renal cell cancer (355 patients), 4 hepatocellular carcinoma (371 patients), 4 thyroid cancer (110 patients) and 1 each for lung cancer (114 patients) and nasopharynx cancer (87 patients) (Fig. 2a). 
Table 2 Summary of included studies

\begin{tabular}{|c|c|c|c|c|c|c|c|c|c|c|c|c|}
\hline \multirow[t]{2}{*}{ Author } & \multicolumn{6}{|c|}{ Character of studies } & \multicolumn{6}{|c|}{ Character of patients } \\
\hline & Year & $\begin{array}{l}\text { Study } \\
\text { period }\end{array}$ & Study design & Country & Follow-up & $\begin{array}{l}\text { NOS } \\
\text { (Stars) }\end{array}$ & $\begin{array}{l}\text { Primary } \\
\text { tumor }\end{array}$ & Case & $\begin{array}{l}\text { Case } \\
\text { with } \\
\text { MSCC }\end{array}$ & $\begin{array}{l}\text { Male } \\
(\%)\end{array}$ & Age & $\begin{array}{l}\text { Overall } \\
\text { survival } \\
\text { (median/ } \\
\text { mean) }\end{array}$ \\
\hline van der Linden [9] & 2005 & 1996-1998 & $\begin{array}{l}\text { retrospective } \\
\text { cohort }\end{array}$ & Netherlands & $\begin{array}{l}\leq 32 \mathrm{~m} \text { or } \\
\text { until death }\end{array}$ & 8 & $\mathrm{Nl}$ & 342 & 12 & 53 & mean: 66 & median:7 m \\
\hline Patchell [23] & 2005 & 1992-2002 & $\begin{array}{l}\text { marched-pair } \\
\text { study }\end{array}$ & USA & $\begin{array}{l}\text { Median: } \\
\text { surgery } \\
\text { group: } 3.4 \text { m; } \\
\text { radiation } \\
\text { group: } 3.1 \text { m }\end{array}$ & 9 & $\mathrm{Nl}$ & 101 & 101 & 70 & median: 60 & NS \\
\hline Chen [24] & 2007 & 2000-2005 & $\begin{array}{l}\text { retrospective } \\
\text { cohort }\end{array}$ & China & NS & 8 & NSCLC & 31 & 31 & 61 & mean: 61.4 & $\begin{array}{l}\text { median:8.8 } \\
\text { m }\end{array}$ \\
\hline Leithner [25] & 2008 & 1998-2006 & $\begin{array}{l}\text { prospective+ } \\
\text { retrospective } \\
\text { cohort }\end{array}$ & Austria & $\geq 12 \mathrm{~m}$ & 7 & $\mathrm{Nl}$ & 69 & NS & 54 & mean: 60 & $\begin{array}{l}\text { median:14 } \\
\text { m }\end{array}$ \\
\hline Park [26] & 2011 & $2001-2008$ & $\begin{array}{l}\text { retrospective } \\
\text { cohort }\end{array}$ & Korea & Mean: $25.8 \mathrm{~m}$ & 8 & $\mathrm{Nl}$ & 103 & 103 & 62 & mean:54.6 & $\begin{array}{l}\text { median:10 } \\
\mathrm{m}\end{array}$ \\
\hline Arrigo [27] & 2011 & 1999-2009 & $\begin{array}{l}\text { retrospective } \\
\text { cohort }\end{array}$ & USA & NS & 9 & $\mathrm{Nl}$ & 200 & 172 & 61 & mean: 58.9 & median:8 m \\
\hline Rades [28] & 2012 & 1992-2010 & $\begin{array}{l}\text { retrospective } \\
\text { cohort }\end{array}$ & Germany & NS & 8 & NSCLC & 356 & 356 & 74 & median:64 & NS \\
\hline Crnalic [29] & 2012 & 2003-2010 & $\begin{array}{l}\text { retrospective } \\
\text { cohort }\end{array}$ & Sweden & NS & 7 & PCa & 68 & 68 & 100 & median:71 & NS \\
\hline Chong [30] & 2012 & 2002-2010 & $\begin{array}{l}\text { retrospective } \\
\text { cohort }\end{array}$ & Korea & NS & 8 & $\mathrm{NI}$ & 105 & 105 & 69 & mean:58.3 & median:6 m \\
\hline Rades [31] & 2013 & 1992-2011 & $\begin{array}{l}\text { retrospective } \\
\text { cohort }\end{array}$ & Germany & NS & 8 & $\mathrm{NI}$ & 2029 & 2029 & NS & NS & NS \\
\hline Ju [32] & 2013 & $2002-2011$ & $\begin{array}{l}\text { retrospective } \\
\text { cohort }\end{array}$ & USA & NS & 8 & PCa & 27 & 27 & 100 & median: 65 & $\begin{array}{l}\text { median:10.2 } \\
\mathrm{m}\end{array}$ \\
\hline Bakker [33] & 2014 & 2006-2013 & $\begin{array}{l}\text { retrospective } \\
\text { cohort }\end{array}$ & Netherlands & NS & 6 & $\mathrm{RCC}$ & 21 & NS & NS & NS & $\begin{array}{l}\text { median: } 25 \\
\mathrm{~m}\end{array}$ \\
\hline Bollen [34] & 2014 & $2001-2010$ & $\begin{array}{l}\text { retrospective } \\
\text { cohort }\end{array}$ & Netherlands & Median: 6.6y & 9 & $\mathrm{Nl}$ & 1043 & NS & 52 & mean:64.8 & $\begin{array}{l}\text { median:4.8 } \\
\text { m }\end{array}$ \\
\hline Vanek [35] & 2015 & 2006-2012 & $\begin{array}{l}\text { retrospective } \\
\text { cohort }\end{array}$ & Czech & NS & 8 & $\mathrm{Nl}$ & 166 & 166 & NS & mean:62 & $\begin{array}{l}\text { median:16 } \\
\text { m }\end{array}$ \\
\hline Tang [36] & 2015 & 2002-2013 & $\begin{array}{l}\text { retrospective } \\
\text { cohort }\end{array}$ & China & $\begin{array}{l}\text { Median: } 13.5 \\
\text { m }\end{array}$ & 9 & NSCLC & 116 & 116 & 65 & median: 55 & NS \\
\hline Lei [18] & 2015 & 2005-2015 & $\begin{array}{l}\text { retrospective } \\
\text { cohort }\end{array}$ & China & Mean: $9.7 \mathrm{~m}$ & 9 & NSCLC & 64 & 64 & 66 & median:57 & $\begin{array}{l}\text { median:6.3 } \\
\text { m }\end{array}$ \\
\hline Chen [37] & 2015 & $2000-2010$ & $\begin{array}{l}\text { retrospective } \\
\text { cohort }\end{array}$ & China & NS & 8 & NSCLC & 50 & 50 & 68 & mean: 61.6 & $\begin{array}{l}\text { median:7.5 } \\
\text { m }\end{array}$ \\
\hline Meng [38] & 2016 & $2002-2012$ & $\begin{array}{l}\text { retrospective } \\
\text { cohort }\end{array}$ & China & NS & 7 & $\mathrm{PCa}$ & 29 & NS & 100 & median: 71 & $\begin{array}{l}\text { median: } 44 \\
\text { m }\end{array}$ \\
\hline Park [39] & 2016 & 2010-2014 & $\begin{array}{l}\text { prospective } \\
\text { cohort }\end{array}$ & Korea & NS & 8 & NSCLC & 50 & 50 & 54 & mean: 58.0 & $\begin{array}{l}\text { median:5.2 } \\
\text { m }\end{array}$ \\
\hline Huddart [40] & 1997 & 1984-1992 & $\begin{array}{l}\text { retrospective } \\
\text { cohort }\end{array}$ & UK & NS & 8 & $\mathrm{PCa}$ & 69 & 69 & 100 & NS & $\begin{array}{l}\text { median: } 3.8 \\
\text { m }\end{array}$ \\
\hline North [8] & 2005 & NS & $\begin{array}{l}\text { retrospective } \\
\text { cohort }\end{array}$ & USA & NS & 9 & $\mathrm{NI}$ & 61 & NS & 56 & mean: 52.4 & $\begin{array}{l}\text { median:10 } \\
\text { m }\end{array}$ \\
\hline Williams [41] & 2009 & 1993-2005 & $\begin{array}{l}\text { retrospective } \\
\text { cohort }\end{array}$ & USA & NS & 9 & PCa & 44 & NS & 100 & median:68 & $\begin{array}{l}\text { median:5.4 } \\
\text { m }\end{array}$ \\
\hline Rades [42] & 2012 & 1992-2010 & $\begin{array}{l}\text { retrospective } \\
\text { cohort }\end{array}$ & Germany & NS & 7 & $\mathrm{PCa}$ & 218 & 218 & 100 & NS & NS \\
\hline
\end{tabular}


Table 2 Summary of included studies (Continued)

\begin{tabular}{|c|c|c|c|c|c|c|c|c|c|c|c|c|}
\hline \multirow[t]{2}{*}{ Author } & \multicolumn{6}{|c|}{ Character of studies } & \multicolumn{6}{|c|}{ Character of patients } \\
\hline & Year & $\begin{array}{l}\text { Study } \\
\text { period }\end{array}$ & Study design & Country & Follow-up & $\begin{array}{l}\text { NOS } \\
\text { (Stars) }\end{array}$ & $\begin{array}{l}\text { Primary } \\
\text { tumor }\end{array}$ & Case & $\begin{array}{l}\text { Case } \\
\text { with } \\
\text { MSCC }\end{array}$ & $\begin{array}{l}\text { Male } \\
(\%)\end{array}$ & Age & $\begin{array}{l}\text { Overall } \\
\text { survival } \\
\text { (median/ } \\
\text { mean) }\end{array}$ \\
\hline Crnalic [43] & 2012 & $2003-2008$ & $\begin{array}{l}\text { retrospective } \\
\text { cohort }\end{array}$ & Sweden & $\begin{array}{l}\text { Median: } \\
\text { naïve: } 26 \text { m; } \\
\text { refractory: } 12 \\
\mathrm{~m}\end{array}$ & 7 & PCa & 54 & 54 & 100 & NS & NS \\
\hline Lee $[44]$ & 2014 & $2005-2010$ & $\begin{array}{l}\text { retrospective } \\
\text { cohort }\end{array}$ & Korea & NS & 7 & $\mathrm{NI}$ & 200 & NS & 59 & mean: 59.9 & $\begin{array}{l}\text { mean: } 10.8 \\
\mathrm{~m}\end{array}$ \\
\hline Sellin [45] & 2015 & 1993-2010 & $\begin{array}{l}\text { retrospective } \\
\text { cohort }\end{array}$ & USA & NS & 9 & $\mathrm{TCa}$ & 43 & NS & 60 & NS & $\begin{array}{l}\text { median:15.4 } \\
\text { m }\end{array}$ \\
\hline Drzymalski [46] & 2010 & 1990-2009 & $\begin{array}{l}\text { retrospective } \\
\text { cohort }\end{array}$ & USA & NS & 8 & PCa & 333 & 77 & 100 & median: 68 & $\begin{array}{l}\text { median:24 } \\
\mathrm{m}\end{array}$ \\
\hline Tancioni [47] & 2012 & 2004-2007 & $\begin{array}{l}\text { retrospective } \\
\text { cohort }\end{array}$ & Italy & NS & 9 & $\mathrm{NI}$ & 151 & 151 & 51 & median: 62 & $\begin{array}{l}\text { median:14 } \\
\text { m }\end{array}$ \\
\hline Tatsui [48] & 2014 & 1993-2007 & $\begin{array}{l}\text { retrospective } \\
\text { cohort }\end{array}$ & USA & $\begin{array}{l}\text { Median: } 77.9 \\
\mathrm{~m}\end{array}$ & 9 & $\mathrm{RCC}$ & 267 & 267 & 77 & median:59.2 & $\begin{array}{l}\text { median:11.3 } \\
\text { m }\end{array}$ \\
\hline Petteys [49] & 2016 & $2000-2011$ & $\begin{array}{l}\text { retrospective } \\
\text { cohort }\end{array}$ & USA & NS & 8 & RCC & 30 & NS & 77 & mean:57.6 & $\begin{array}{l}\text { median:11.4 } \\
\text { m }\end{array}$ \\
\hline Rades [50] & 2016 & NS & $\begin{array}{l}\text { retrospective } \\
\text { cohort }\end{array}$ & Germany & Median:6.5 m & 7 & $\mathrm{TCa}$ & 14 & 14 & 29 & median:70 & NS \\
\hline Kato [51] & 2016 & 1984-2011 & $\begin{array}{l}\text { retrospective } \\
\text { cohort }\end{array}$ & Japan & NS & 7 & $\mathrm{TCa}$ & 32 & NS & 22 & mean:60.6 & median:6.4y \\
\hline Sciubba [52] & 2007 & 1993-2001 & $\begin{array}{l}\text { retrospective } \\
\text { cohort }\end{array}$ & USA & Median: 13 m & 9 & $\mathrm{BCa}$ & 87 & NS & 0 & median: 53 & $\begin{array}{l}\text { median: } 21 \\
\text { m }\end{array}$ \\
\hline Walcott [53] & 2011 & $2001-2009$ & $\begin{array}{l}\text { retrospective } \\
\text { cohort }\end{array}$ & USA & NS & 7 & $\mathrm{BCa}$ & 15 & 15 & 0 & median: 58 & $\begin{array}{l}\text { median: } \\
34.2 \mathrm{~m}\end{array}$ \\
\hline Tancioni [54] & 2011 & 2004-2009 & $\begin{array}{l}\text { retrospective } \\
\text { cohort }\end{array}$ & Italy & Median:26 m & 8 & $\mathrm{BCa}$ & 23 & 23 & 0 & median:55 & $\begin{array}{l}\text { median:36 } \\
\text { m }\end{array}$ \\
\hline Zadnik [55] & 2014 & 2002-2011 & $\begin{array}{l}\text { retrospective } \\
\text { cohort }\end{array}$ & USA & $\begin{array}{l}\text { Median:18.3 } \\
\text { m }\end{array}$ & 8 & $\mathrm{BCa}$ & 43 & NS & 0 & median: 56 & $\begin{array}{l}\text { median:26.8 } \\
\text { m }\end{array}$ \\
\hline Ulmar [56] & 2007 & 1984-2005 & $\begin{array}{l}\text { retrospective } \\
\text { cohort }\end{array}$ & Germany & NS & 6 & $\mathrm{RCC}$ & 37 & 20 & 84 & median:64 & $\begin{array}{l}\text { mean:13.7 } \\
\mathrm{m}\end{array}$ \\
\hline Jiang [57] & 2014 & 1999-2013 & $\begin{array}{l}\text { retrospective } \\
\text { cohort }\end{array}$ & China & Mean:42.7 m & 7 & $\mathrm{TCa}$ & 21 & NS & 24 & mean:62 & NS \\
\hline Oliveira [58] & 2015 & 2010-2013 & $\begin{array}{l}\text { retrospective } \\
\text { cohort }\end{array}$ & Brazil & mean: 13.8 m & 7 & $\mathrm{NI}$ & 68 & 45 & 66 & mean:62.2 & NS \\
\hline Kataoka [59] & 2012 & 1990-2008 & $\begin{array}{l}\text { retrospective } \\
\text { cohort }\end{array}$ & Japan & mean: $21 \mathrm{~m}$ & 9 & $\mathrm{NI}$ & 143 & NS & 64 & median:61 & mean: $22 \mathrm{~m}$ \\
\hline Aoude [60] & 2016 & 2003-2012 & $\begin{array}{l}\text { retrospective } \\
\text { cohort }\end{array}$ & Canada & NS & 7 & $\mathrm{NI}$ & 126 & NS & 44 & mean:59.2 & mean:27 m \\
\hline Bartels [61] & 2007 & 1998-2005 & $\begin{array}{l}\text { retrospective } \\
\text { cohort }\end{array}$ & Netherlands & NS & 7 & $\mathrm{NI}$ & 219 & 185 & 58 & mean:62.7 & median:3 m \\
\hline Lei [62] & 2016 & 2005-2015 & $\begin{array}{l}\text { retrospective } \\
\text { cohort }\end{array}$ & China & mean: 11.5 m & 9 & $\mathrm{NI}$ & 206 & 206 & 51 & median:56 & $\begin{array}{l}\text { median:7.3 } \\
\text { m }\end{array}$ \\
\hline Chang [63] & 2001 & 1981-1997 & $\begin{array}{l}\text { retrospective } \\
\text { cohort }\end{array}$ & China & NS & 7 & $\mathrm{HCC}$ & 102 & NS & 93 & mean:59.2 & median:3 m \\
\hline Chen [64] & 2010 & $2001-2007$ & $\begin{array}{l}\text { retrospective } \\
\text { cohort }\end{array}$ & China & NS & 7 & $\mathrm{HCC}$ & 41 & NS & 78 & mean:53.2 & $\begin{array}{l}\text { mean:10.4 } \\
\text { m }\end{array}$ \\
\hline Choi [65] & 2015 & 1992-2012 & $\begin{array}{l}\text { retrospective } \\
\text { cohort }\end{array}$ & Korea & median:4.2 m & 9 & $\mathrm{HCC}$ & 192 & 25 & 82 & mean:56 & $\begin{array}{l}\text { median:4.5 } \\
\text { m }\end{array}$ \\
\hline Guo [66] & 2003 & 1996-1998 & $\begin{array}{l}\text { retrospective } \\
\text { cohort }\end{array}$ & USA & NS & 6 & $\mathrm{NI}$ & 60 & 60 & NS & NS & $\begin{array}{l}\text { median:4.1 } \\
\text { m }\end{array}$ \\
\hline
\end{tabular}


Table 2 Summary of included studies (Continued)

\begin{tabular}{|c|c|c|c|c|c|c|c|c|c|c|c|c|}
\hline \multirow[t]{2}{*}{ Author } & \multicolumn{6}{|c|}{ Character of studies } & \multicolumn{6}{|c|}{ Character of patients } \\
\hline & Year & $\begin{array}{l}\text { Study } \\
\text { period }\end{array}$ & Study design & Country & Follow-up & $\begin{array}{l}\text { NOS } \\
\text { (Stars) }\end{array}$ & $\begin{array}{l}\text { Primary } \\
\text { tumor }\end{array}$ & Case & $\begin{array}{l}\text { Case } \\
\text { with } \\
\text { MSCC }\end{array}$ & $\begin{array}{l}\text { Male } \\
(\%)\end{array}$ & Age & $\begin{array}{l}\text { Overall } \\
\text { survival } \\
\text { (median/ } \\
\text { mean) }\end{array}$ \\
\hline Moon [67] & 2011 & 1987-2009 & $\begin{array}{l}\text { retrospective } \\
\text { cohort }\end{array}$ & Korea & NS & 6 & $\mathrm{NI}$ & 182 & NS & 61 & median:56 & median:8 m \\
\hline Yang [68] & 2012 & 2001-2009 & $\begin{array}{l}\text { retrospective } \\
\text { cohort }\end{array}$ & Korea & NS & 7 & $\mathrm{NI}$ & 217 & NS & 59 & mean:55.5 & median:6 m \\
\hline $\begin{array}{l}\text { Helweg-Larsen } \\
\text { [69] }\end{array}$ & 2000 & $\begin{array}{l}\text { a period } \\
\text { of } 3.5 \\
\text { years }\end{array}$ & $\begin{array}{l}\text { prospective } \\
\text { cohort }\end{array}$ & Denmark & $\begin{array}{l}\geq 11 \mathrm{~m} \text { or } \\
\text { until death }\end{array}$ & 9 & $\mathrm{NI}$ & 153 & 153 & 51 & NS & $\begin{array}{l}\text { median:3.6 } \\
\text { m }\end{array}$ \\
\hline Kumar [70] & 2014 & $2007-2011$ & $\begin{array}{l}\text { retrospective } \\
\text { cohort }\end{array}$ & Singapore & $\begin{array}{l}\geq 1 y \text { or until } \\
\text { death }\end{array}$ & 9 & NPC & 87 & NS & 78 & median: 52 & $\begin{array}{l}\text { median:13 } \\
\text { m }\end{array}$ \\
\hline Mizumoto [71] & 2008 & $2002-2006$ & $\begin{array}{l}\text { retrospective } \\
\text { cohort }\end{array}$ & Japan & $\begin{array}{l}\geq 1 y \text { or until } \\
\text { death }\end{array}$ & 9 & $\mathrm{NI}$ & 544 & 133 & 53 & median:63 & $\begin{array}{l}\text { median:5.9 } \\
\text { m }\end{array}$ \\
\hline Ogihara [72] & 2006 & 1993-2001 & $\begin{array}{l}\text { retrospective } \\
\text { cohort }\end{array}$ & Japan & NS & 7 & LC & 114 & NS & 61 & mean:64.6 & mean: $6.2 \mathrm{~m}$ \\
\hline Pointillart [11] & 2011 & 2005-2007 & $\begin{array}{l}\text { prospective } \\
\text { cohort }\end{array}$ & France & $\begin{array}{l}\geq 1 y \text { or until } \\
\text { death }\end{array}$ & 8 & $\mathrm{NI}$ & 142 & NS & 57 & mean:61.8 & median:5 m \\
\hline Rades [73] & 2006 & 1992-2003 & $\begin{array}{l}\text { retrospective } \\
\text { cohort }\end{array}$ & Germany & NS & 7 & $\mathrm{BCa}$ & 335 & 335 & 0 & NS & $\begin{array}{l}\text { median:20 } \\
\text { m }\end{array}$ \\
\hline Switlyk [76] & 2015 & $2007-2008$ & $\begin{array}{l}\text { retrospective } \\
\text { cohort }\end{array}$ & Norway & NS & 7 & $\mathrm{NI}$ & 173 & 47 & 56 & median:65 & $\begin{array}{l}\text { median:8.2 } \\
\mathrm{m}\end{array}$ \\
\hline Tao [74] & 2004 & 1992-2002 & $\begin{array}{l}\text { retrospective } \\
\text { cohort }\end{array}$ & China & $\geq 6 \mathrm{~m}$ & 9 & $\mathrm{NI}$ & 63 & NS & 59 & mean:52 & mean:6 m \\
\hline Tomita [6] & 2001 & 1987-1991 & $\begin{array}{l}\text { retrospective } \\
\text { cohort }\end{array}$ & Japan & until 1992 & 7 & $\mathrm{NI}$ & 67 & NS & 46 & mean:56.3 & NS \\
\hline Weber [76] & 2013 & NS & $\begin{array}{l}\text { retrospective } \\
\text { cohort }\end{array}$ & Germany & NS & 6 & $\mathrm{BCa}$ & 145 & 145 & 0 & median:63 & NS \\
\hline Yamashita [77] & 2011 & 2006-2008 & $\begin{array}{l}\text { prospective } \\
\text { cohort }\end{array}$ & USA & $\geq 1 y$ & 9 & $\mathrm{NI}$ & 85 & NS & 52 & mean:60.3 & $\begin{array}{l}\text { median:11.6 } \\
\mathrm{m}\end{array}$ \\
\hline Yeung [78] & 2014 & 2000-2010 & $\begin{array}{l}\text { retrospective } \\
\text { cohort }\end{array}$ & China & $\geq 1 y$ & 9 & $\mathrm{NI}$ & 128 & 128 & 71 & $\begin{array}{l}\text { mean:60.2; } \\
\text { median:59 }\end{array}$ & mean:7.6 m \\
\hline Zhang [79] & 2013 & 2003-2011 & $\begin{array}{l}\text { retrospective } \\
\text { cohort }\end{array}$ & China & mean: 15.7 m & 9 & $\mathrm{HCC}$ & 36 & NS & 89 & mean:49.9 & NS \\
\hline Enkaoua [80] & 1997 & NS & $\begin{array}{l}\text { retrospective } \\
\text { cohort }\end{array}$ & France & NS & 6 & $\mathrm{NI}$ & 71 & NS & 51 & mean: 59.8 & NS \\
\hline
\end{tabular}

Note: $\mathrm{BCa}=$ breast cancer; $\mathrm{HCC}=$ hepatocellular carcinoma; $\mathrm{LC}=$ lung cancer; $\mathrm{MSCC}=$ metastatic spinal cord compression; $\mathrm{NI}=$ not identified; $\mathrm{NOS}=$ The NewcastleOttawa Scale; NPC = nasopharyngeal carcinoma; NS = not specified; NSCLC = non-small cell lung cancer; $\mathrm{OS} \%=$ percentage of overall survival; $\mathrm{PCa}=$ prostate cancer; $\mathrm{RCC}=$ renal cell cancer; $\mathrm{RT}=$ radiotherapy; SUR = surgery; $\mathrm{TCa}=$ thyroid cancer

\section{Participants' characteristics}

Of the 63 studies eligible for inclusion, 36 reported number of patients with MSCC before treatment, which added up to 5820 in 7212 patients (80.7\%). Apart from 14 studies for prostate and breast cancer, 45 studies reported percentage of gender, with 4169 (59.5\%) males and 2836 (40.5\%) females included. An overall mean age of 4564 patients involved in the 31 studies was 61.9 years. Regarding the location of metastases, data was available in 36 studies containing 4046 patients, and maximum number of patients developed thoracic metastasis, followed by lumbar, cervical, thoracolumbar, diffused, cervicothoracic, lumbosarcral and sacrum metastasis (Fig. 2b).

\section{Therapeutic modality}

Modality of therapy was available in 61 articles containing 10,004 patients (Fig. 2c). Patients predominantly received surgery or radiotherapy as major treatments. Surgery types mainly included 3324 decompression surgery with/without instrumented procedures, 108 total en bloc spondylectomy, 323 spinal fusion. Radiotherapy was performed in 5981 patients as major treatment. Other treatments, such as adjuvant therapies, radiotherapy, 

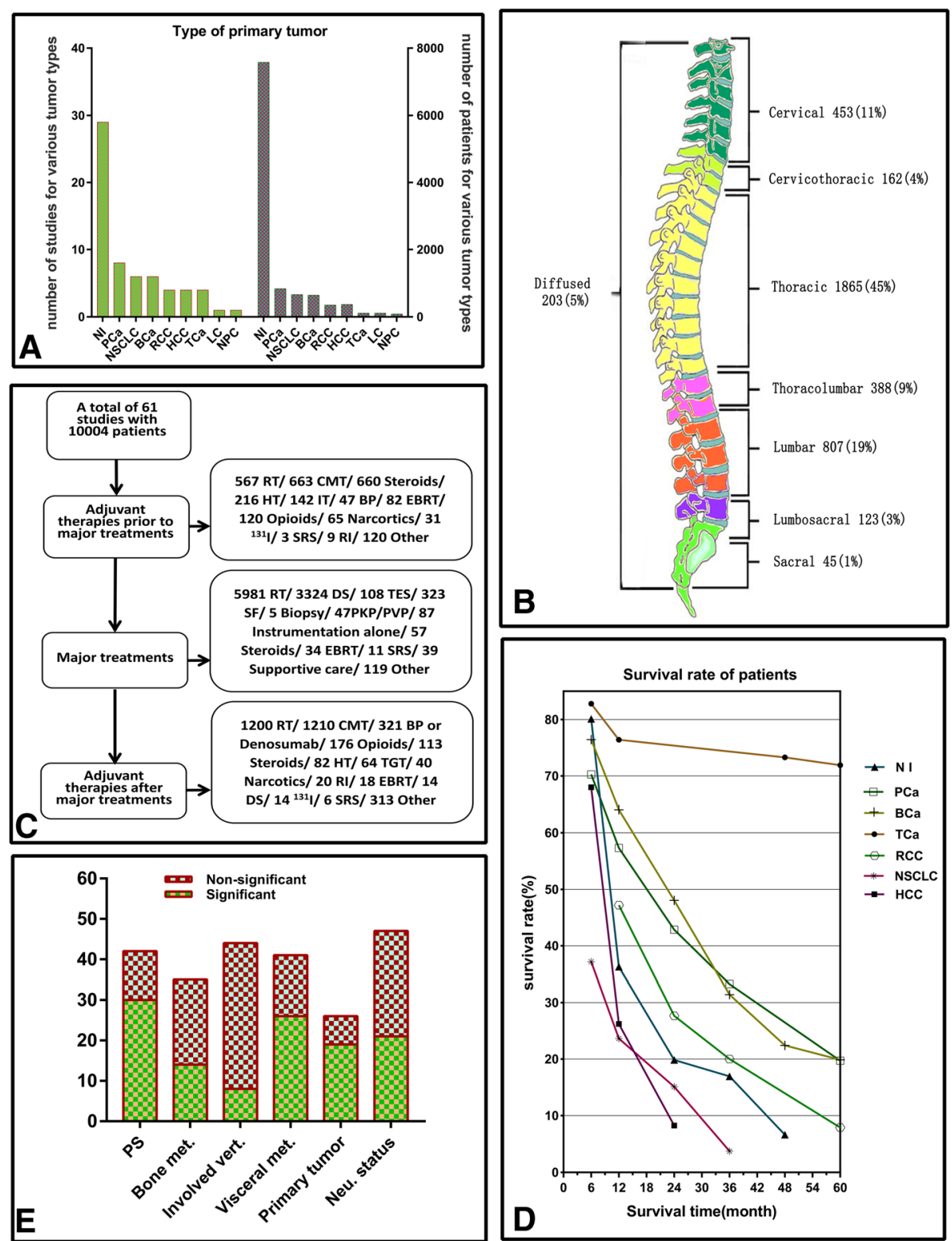

Fig. 2 a Number of studies and patients for each type of primary tumor; b Distribution of spinal metastatic location; diffused patients include those presented with three or four sections of spinal metastases; c Therapeutic modalities provided for patients; $\mathbf{d}$ Overall survival rate for primary tumor; e Prognostic effect of factors included in revised Tokuhashi Score. (Note: NI = not identified; PCa = prostate cancer; NSCLC = non-small cell lung cancer; $\mathrm{BCa}=$ breast cancer; $\mathrm{RCC}=$ renal cell cancer; $\mathrm{HCC}=$ hepatocellular carcinoma; $\mathrm{TCa}=$ thyroid cancer; $\mathrm{LC}=$ lung cancer; $\mathrm{NPC}=$ nasopharyngeal carcinoma; $\mathrm{RT}=$ radiotherapy; $\mathrm{CMT}=$ chemotherapy; $\mathrm{HT}=$ hormonal therapy; $\mathrm{IT}=$ immunotherapy; $\mathrm{BP}=$ bisphosphonates; $\mathrm{EBRT}=$ external-beam radiation therapy; SRS = stereotactic radiosurgery; RI = radioisotopes; DS = decompression surgery; SF = spinal fusion; $\mathrm{TGT}=$ targeted therapy; PS = performance status; met. = metastases; Neu. = neurological)

chemotherapy, targeted therapy, immunotherapy, bisphosphonates, were provided alone or with various combination prior to or after major procedures.

\section{Follow-up and overall survival}

Data of follow-up was available in 27 studies, and 7 of them were followed for more than one year or until death. 7 were followed for an average period ranged 9.7-42.7 months and 10 were followed for a median period ranged 3.1-79.2 months. After treatment, the average survival time was ranged 6-27 months, and median survival time was ranged 3-77 months as reported in 8 and 42 studies respectively. Survival rates at $6,12,24,36,48$ and 60 months for various types of primary tumors were calculated and presented in 
Fig. 2d. Overall, thyroid cancer had the highest survival rate, followed by prostate cancer/ breast cancer, renal cell cancer and mixed cancer, and non-small cell lung cancer and hepatocellular carcinoma lived for the shorted life span.

\section{Qualitative data summary on prognostic factors}

Numbers of studies that showe significance and non-significance for each prognostic factor are presented in Fig. 2e. Performance status was analyzed in 42 articles and $30(71.4 \%)$ supported it as a significant factor. Prediction value of bone metastasis was involved in 35 studies, and $14(40.0 \%)$ reported statistical significance. Number of involved vertebrae was analyzed in 44 studies, and $8(18.2 \%)$ studies drew significant conclusions. As for visceral metastasis, 26 (63.4\%) studies regarded it as a significant predictor in 41 involved studies. Totally, 26 studies analyzed the influence of primary tumor on survival, and 19 (73.1\%) of them were ofstatistical sig nificance. Neurological status was involved in 47 studies and 21 (44.7\%) were statistically significant.

\section{Quantitative data synthesis}

Prognostic effects of five factors (primary tumor type was not included for lack of homogeneous comparison between groups) were identified. The results of meta-analyses are presented in Table 3. As shown in these results, patients with 'severe' disability (KPS 1040) and 'moderate' disability (KPS 50-70) have similar survival rates $(\mathrm{HR}=1.27$, CI 95\% 0.89-1.79, $P=0.186$ ) and both groups are worse than patients with no to mild disability (KPS 80-100) (Fig. 3a). And patients with 3 or more involved vertebrae have worse survival than patients with 1-2 involved vertebrae, while patients with single and multiple involved vertebrae have similar survival rates $(\mathrm{HR}=1.22$, CI 95\% 0.96-1.56, $P=0.102)$ (Fig. $3 \mathrm{c})$. All the other comparisons between various groups of patients for the five prognostic factors were proved to be significant (Fig. 3a-e). All the meta-analyses were performed with a fixed-effect model except comparison between ambulation and non-ambulation $\left(\mathrm{I}^{2}=52.8 \%\right)$. Egger's test for number of involved vertebrae (1-2 VS. $\geq 3)$ presented a significant publication bias $(P=0.046)$ and a nonparametric trim and fill method was performed to rectify the detected publication bias (Fig. 3f). Pooled effect size of HR was 1.24 (CI 95\% 1.10 1.40, $P=0.001$ ) after 3 studies were filled.

According to these results, remodifications on the cut-off of KPS and number of involved vertebrae were conducted for the RTS, and a remodified version of RTS

Table 3 Results of quantitative meta-analyses

\begin{tabular}{|c|c|c|c|c|c|c|c|c|c|c|}
\hline \multirow[t]{2}{*}{ Prognostic factor } & \multirow[t]{2}{*}{$\begin{array}{l}\text { No. of } \\
\text { studies }\end{array}$} & \multirow[t]{2}{*}{$\begin{array}{l}\text { No. of } \\
\text { patients }\end{array}$} & \multirow[t]{2}{*}{$\begin{array}{l}\text { Pooled } \\
\text { effect } \\
\text { size }(H R)\end{array}$} & \multirow[t]{2}{*}{$\begin{array}{l}\mathrm{Cl} \\
95 \%\end{array}$} & \multirow[t]{2}{*}{$\begin{array}{l}P^{2} \\
(\%)\end{array}$} & \multirow[t]{2}{*}{$\begin{array}{l}\text { Effect } \\
\text { model }\end{array}$} & \multirow[t]{2}{*}{$\begin{array}{l}Z \text { test } \\
(P \text { value })\end{array}$} & \multirow{2}{*}{$\begin{array}{l}\text { Excluded } \\
\text { studies by } \\
\text { sensitivity } \\
\text { analysis }\end{array}$} & \multicolumn{2}{|c|}{$\begin{array}{l}\text { Publication } \\
\text { bias } \\
(P \text { value })\end{array}$} \\
\hline & & & & & & & & & Begg's & $\overline{\text { Egger's }}$ \\
\hline KPS(10-40VS.50-70) $[9,38,71]$ & 3 & 479 & 1.27 & $\begin{array}{l}(0.89 \\
1.79)\end{array}$ & 19.8 & Fixed & $0.186^{a}$ & 0 & 1.000 & 0.188 \\
\hline KPS(10-40VS.80-100) [11, 26, 38, 76] & 4 & 377 & 3.46 & $\begin{array}{l}(1.83 \\
6.57)\end{array}$ & 0.0 & Fixed & $<0.001$ & $3[9,71,79]$ & 0.308 & 0.404 \\
\hline $\begin{array}{l}\text { KPS(50-70VS.80-100) } \\
{[26,75,78,79]}\end{array}$ & 4 & 455 & 2.47 & $\begin{array}{l}(1.83, \\
3.32)\end{array}$ & 0.0 & Fixed & $<0.001$ & 0 & 1.000 & 0.834 \\
\hline KPS(10-70VS.80-100) [30, 31, 32-35, 46] & 6 & 1307 & 1.94 & $\begin{array}{l}(1.68 \\
2.25)\end{array}$ & 7.0 & Fixed & $<0.001$ & 0 & 0.133 & 0.214 \\
\hline ECOG(1-2VS.3-4) $[19,37,40,43,64,66,75]$ & 7 & 887 & 2.22 & $\begin{array}{l}(1.82, \\
2.71)\end{array}$ & 23.0 & Fixed & $<0.001$ & $4[29,32,60,72]$ & 0.548 & 0.345 \\
\hline $\begin{array}{l}\text { Extraspinal bone metastases }[9,19,26,29,32,34, \\
38,43,47,60,70]\end{array}$ & 11 & 3831 & 1.37 & $\begin{array}{l}(1.23, \\
1.52)\end{array}$ & 38.5 & Fixed & $<0.001$ & 0 & 0.755 & 0.819 \\
\hline $\begin{array}{l}\text { No. of involved vertebrae ( } \geq 2 \text { VS.1) }[26,34,37,41 \text {, } \\
52,60]\end{array}$ & 6 & 450 & 1.22 & $\begin{array}{l}(0.96 \\
1.56)\end{array}$ & 31.9 & Fixed & $0.102^{\mathrm{a}}$ & 0 & 1.000 & 0.434 \\
\hline $\begin{array}{l}\text { No. of involved vertebrae ( } \geq 3 \vee S .1-2)[8,19,29,31 \text {, } \\
38,43,53,63,75]\end{array}$ & 9 & 1292 & 1.34 & $\begin{array}{l}(1.17 \\
1.53)\end{array}$ & 29.7 & Fixed & $<0.001$ & 0 & 0.118 & $0.046^{\mathrm{b}}$ \\
\hline $\begin{array}{l}\text { Visceral metastases }[9,19,26,30,31,33,34,38,44, \\
46,47,52,53,56,58,60,66,76]\end{array}$ & 18 & 1779 & 1.83 & $\begin{array}{l}(1.59 \\
2.09)\end{array}$ & 43.9 & Fixed & $<0.001$ & $\begin{array}{l}7[28,29,32,33 \\
35,43,74,72]\end{array}$ & 0.880 & 0.969 \\
\hline $\begin{array}{l}\text { Ambulatory status }[8,19,26,28-32,36,37,41,43, \\
51,53,60,63,69,71,75]\end{array}$ & 20 & 4456 & 1.80 & $\begin{array}{l}(1.52, \\
2.13)\end{array}$ & 52.8 & Random & $<0.001$ & 0 & 0.922 & 0.953 \\
\hline Frankel (C-D VS. E) $[34,46,49,53,76]$ & 6 & 631 & 1.41 & $\begin{array}{l}(1.10 \\
1.81)\end{array}$ & 39.5 & Fixed & 0.006 & 0 & 0.707 & 0.967 \\
\hline
\end{tabular}

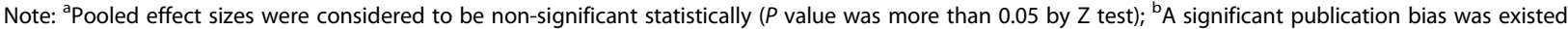
according to Egger's test and the nonparametric trim and fill method was performed to rectify the bias 

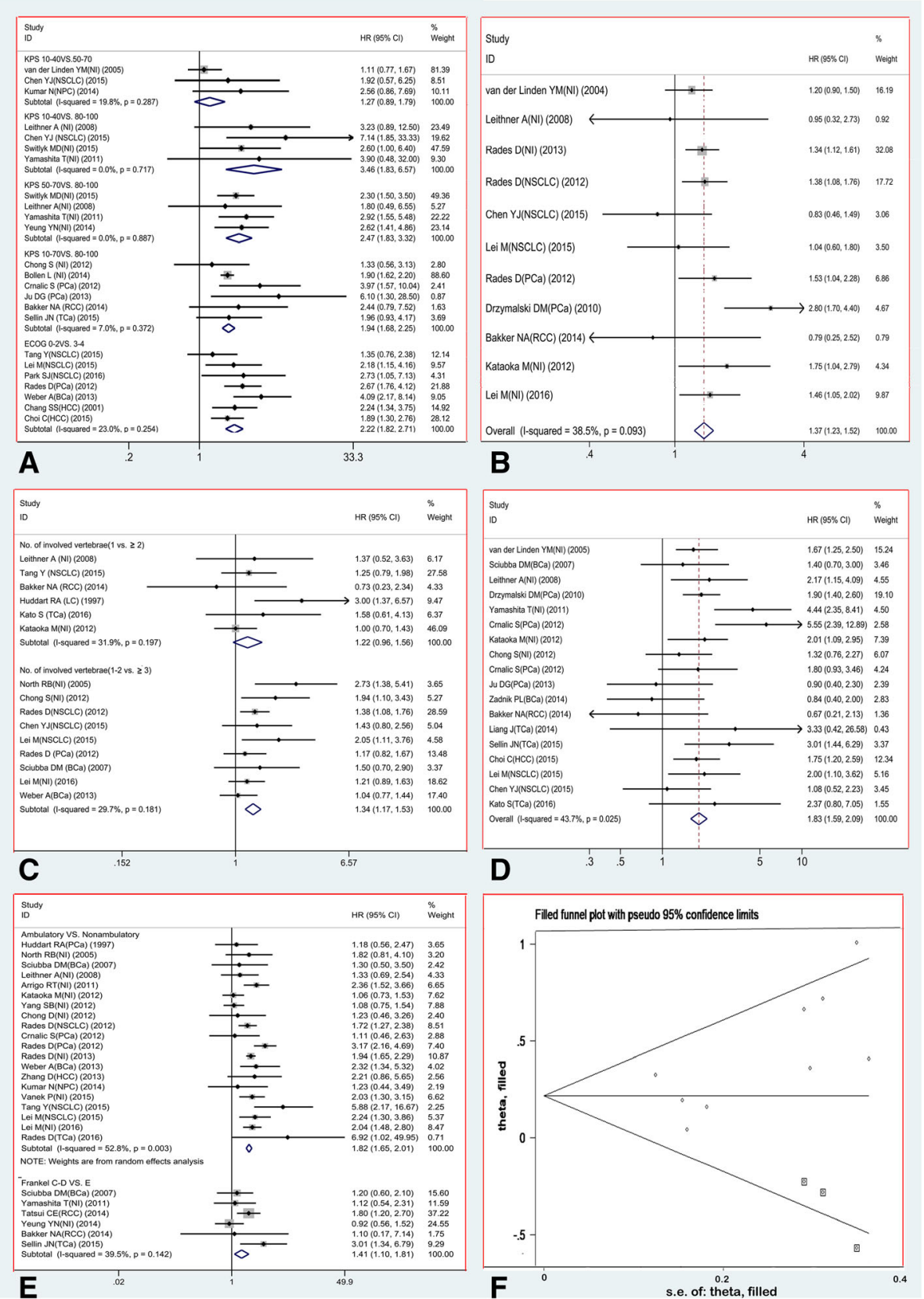

Fig. 3 a Forest plots for effect size of performance status (KPS/ ECOG); b Forest plot for effect size of arising of other bone metastasis; $\mathbf{c}$ Forest plot for effect size of number of involved vertebrae; $\mathbf{d}$ Forest plot for effect size of arising of visceral metastasis; e Forest plot for effect size of neurological status; $\mathbf{f}$ Funnel plot after 3 studies were filled by a nonparametric trim and fill method (the diamonds represent studies which were filled)

is shown in Table 4. Patients with KPS $10-40 / 50-70$ and patients with single/double involved vertebrae were merged together and the total score of the RTS was not changed which was added up to 15 .

\section{Discussion}

The primary aim of the treatment on spinal metastasis is to attain the optimal relief on symptoms of MSCC (e.g. intractable pain and neurological deficit), restore or maintain of spinal stability and improving the quality of life by various individualized therapeutic options. A number of prognostic scoring systems have been established to assist clinicians in predicting prognosis, such as Tokuhashi $[3,4]$, Tomita [6] and Enkaoua [82]. To achieve the optimal remission of symptoms, surgeons must consider patients' life expectancy. However, most of the scores present sources of 
Table 4 A remodified Version of Revised Tokuhashi Score System

\begin{tabular}{|c|c|}
\hline Factors & Score \\
\hline \multicolumn{2}{|l|}{ General condition (Karnofsky Performance Status, KPS) ${ }^{a}$} \\
\hline Poor and moderate (KPS 10-70) & 0 \\
\hline Good (KPS 80-100) & 2 \\
\hline \multicolumn{2}{|l|}{ Extraspinal bone metastases } \\
\hline$\geq 3$ & 0 \\
\hline $1-2$ & 1 \\
\hline 0 & 2 \\
\hline \multicolumn{2}{|l|}{ No. of metastases in the vertebral body ${ }^{\mathrm{b}}$} \\
\hline$\geq 2$ & 0 \\
\hline 1 & 2 \\
\hline \multicolumn{2}{|l|}{ Metastases to the major internal organs } \\
\hline Unremovable & 0 \\
\hline Removable & 1 \\
\hline No metastases & 2 \\
\hline \multicolumn{2}{|l|}{ Primary site of the cancer } \\
\hline Lung, osteosarcoma, stomach, bladder, esophagus, pancreas & 0 \\
\hline Liver, gallbladder, unidentified & 1 \\
\hline Others & 2 \\
\hline Kidney, uterus & 3 \\
\hline Rectum & 4 \\
\hline Thyroid, breast, prostate, carcinoid tumor & 5 \\
\hline \multicolumn{2}{|l|}{ Neurological Status } \\
\hline Complete (Frankel A, B) & 0 \\
\hline Incomplete (Frankel C, D) & 1 \\
\hline None (Frankel E) & 2 \\
\hline
\end{tabular}

Note: This remodified version of RTS was raised according to results in the meta-analyses and remodifications on the cut-off of KPS $\left(^{\mathrm{a}}\right)$ and number of involved vertebrae $\left({ }^{b}\right)$ were conducted for the scoring system. The patients with KPS 10-40/ 50-70 and patients with single/double involved vertebrae were merged together

bias in patient selection and involve conflicting factors. According to RTS, performance status, bone metastasis, number of involved vertebrae, visceral metastasis, primary tumor and spinal cord palsy are significant to predict patients' overall survival $[3,4]$. Current study identified the role of factors included in RTS in predicting overall survival in patients with spinal metastases.

\section{Prognostic effect of factors \\ General condition}

Rades [43] compared overall survival of patients with Eastern Cooperative Oncology Group (ECOG) performance status 1-2 and 3-4, and the former group was presented with a significant higher survival. Van der Linden [9] and Bartels [62] also included performance status in their prognostic scores. Generally, patients with better performance status could tolerate more invasive therapeutic modalities, which would extend patients' survival. However, some other studies did not considered performance status as a significant predictor. Leithner [26] supposed some other factors, such as arising of visceral metastasis and sever neurological deficit, would also make patients debilitated, and further decreased patients' performance status, but these patients might be favourable in otherwise general condition to tolerate invasive therapy. In current study, performance status was identified to be a significant predictor for all except comparison between KPS 10-40 and $50-70(P=0.186)$. Thus, in general, performance status could be identified to be a reliable predictor. Similar to the results of the previous studies $[9,38,71]$, we thought that the cut-off of KPS should not included KPS 10-40/ 50-70 as patients were both too debilitated to be cured from invasive therapies.

\section{Extraspinal bone metastases and number of involved vertebrae}

Rades [32] found that bone metastasis was significant in predicting prognosis of patients treated with radiotherapy. In study of Chong [31], patients with $\leq 2$ column involved had a significant longer overall survival than the ones with $>2$ column involved. Generally, the two factors were often related to biological behaviour of invasion, spread and proliferation, which indicates advanced stages of cancer. In addition, added number of involved vertebrae would increase the difficulty of treatment and probability of occurrence of complications. Meanwhile, many studies presented non-significant results on prognosis effect of the two factors, such as van der Linden [9]. And Tomita Score adopted bone metastasis but not number of spinal metastases [6]. In current study, extraspinal bone metastases and number of involved vertebrae ( $\geq 3$ VS. $1-2$ ) were confirmed to be significant factors, but number of involved vertebrae (multiple VS. single) was of non-significance. Overall, we think that the two factors are reliable but the cut-off of number of involved vertebrae should not included single/ multiple spinal metastases, and use of $>1$ vertebrae as cutoff is less effective for predicting survival than use of $>2$ vertebrae.

\section{Visceral metastases}

In scores of Tomita [6], van der Linden [9] and Enkaoua [82], visceral metastasis is included as a predictor. Rades [29] found that not only arising of visceral metastases with $\geq 2$ sites had a poorer prognosis than arising of $0-1$ site, patients with and without metastasis also had a diverse survival. Generally, visceral metastases is considered as a significant factor due to 3 reasons: (1) it is often related to an advanced stage of cancer; (2) it may increase number of complications; (3) it deliver more metastatic burden to patients than spinal metastasis. However, Bollen [35] found that visceral metastasis was not a significant factor for all but patients with favourable primary tumor types, and 
patients with moderate and unfavourable profile of primary tumors were of very poor prognosis that prognostic effects of visceral metastases were weakened. Regardless of existed controversies, our meta-analysis identified visceral metastases as a significant predictor $(P<0.001)$.

\section{Histology of primary tumor}

As reported by Arrigo [28], primary tumor was a robust predictor in spinal metastasis. Yeung [80] also found that primary tumor types by RTS was a significant predictor overall. Nevertheless, a minority of studies presented a non-significance on the prognostic effect of primary tumor $[19,31,36]$. As reported in study of Lee [45], discrepancy of survival among different primary tumors were not significant. And they insisted that it's due to some advanced adjuvant therapeutic modalities that make patients with primary tumor of high malignancy lived a longer survival. In current study, we figured that thyroid cancer had the highest survival rate, followed by prostate/ breast cancer, renal cell cancer and mixed cancer, and non-small cell lung cancer and hepatocellular carcinoma lived for the shorted life span, which was in accordance with RTS [4].

\section{Neurological status}

Sioutos [5] and Enkaoua [82] included neurological deficit in their scores. Rades [22] and Tang [37] also accepted ambulatory status as a significant factor, since patients with neurological deficit might become too deteriorated to tolerate more aggressive surgical procedures and adjuvant therapies, and more severe complications would arise among paraplegic patients. However, there were also many studies that did not adopt neurological status as a predictor based on their cohorts such as Tomita Score [6]. They insisted that neurological deficit could be improved through appropriate treatment, which would bring about a longer survival. Van der Linden [9] speculated that symptom of myeloplegia could just reflect the location and volume of lesions but not the biological behaviour. In current study, both of ambulatory status and arising of neurological deficit before treatment were confirmed to be significant, which was in accordance with RTS [4].

\section{Remodification on the revised Tokuhashi score}

Tokuhashi Scoring was developed for the preoperative evaluation on the prognosis of metastatic spinal tumors and has been used clinically with minor revisions [3, 4]. For the revised score, consistency rate between the predicted prognosis from the criteria of the total scores and the actual survival was proved to be as high as $86.4 \%$ in the 118 patients evaluated prospectively after 1998 [4]. Yamashita [79] identified the relation between the revised Tokuhashi score and actual survival of 85 patients and found that actual survival matched the predicted survival in 67 (79\%) of 85 patients. Thus, RTS was found to be very effective to predict survival. Nevertheless, some studies identified the RTS as a less predictive and practicable prognostic system [10, 83]. Gakhar [83] found that RTS was only significantly accurate in group of patients with expected survival of more than 12 months but not in groups with less than 1 months or between 6 to 12 months. According to current study, in general, factors of RTS were all valuable in predicting survival as many studies had verified $[65,71]$. While more accurate prognosis may be obtained if remodifications were made on the cut-off of KPS and number of involved vertebrae were conducted for the scoring system in future. Considering the results of quantitative pooling, we thought that patients with KPS 10-40/50-70 and patients with single/ double involved vertebrae should be merged together.

Though RTS was proved to be practicable and accurate for predicting the life expectancy of patients with spinal metastasis in plenty of former studies as well as the current study, it was also limited since it had only analyzed the prognostic effect of preoperative characteristics. The RTS has been used for a long term after it was first established in 1990 and revised in 2005. But to our knowledge, the significant predictors for spinal metastasis have been changed over the time, especially after some effective adjuvant interventions, such as target or chemical therapies have been applied to the clinical treatment. The patients' life expectancy have been obviously altered in some specific tumor types in the recent years. For instance, after the introduction of the anti-VEGF antibody Bevacizumab combined with a Cisplatin-containing regimen was used in nonsquamous NSCLC, and the patients' progression-free survival was significantly improved [82]. In the study of Horn et al., [83] it was also demonstrated that Bevacizumab (more than 14 months) significantly improved the overall survival of patients with adenocarcinoma compared standard therapy (10 months). Hence, apart from the factors that has been involved in the RTS, we propose establishing new scores or new revisions on RTS in the future to sufficiently consider the effect of modern therapeutic modalities, which would further increase the accuracy and prognostic capacity on predicting the patients' survival.

\section{Limitations of this study}

Our study nonetheless has limitations. Firstly, primary articles included were published with design of retrospective cohorts dominantly, and only an average value of $7.8 \pm 1.0$ stars for NOS was presented which would cause some potential bias. It may be due to few prospective cohort studies have been carried out till now. Anyhow, majority of studies were of an acceptable quality and none was showed to be with high risk of bias (NOS $\leq 5$ stars). Secondly, the studies included in this work lacked information on either one or more RTS parameter(s) as few studies had completely 
contained and reported the data about each of the parameter, which would lead to an inevitable bias. What's more, current study could only evaluate and verify the prognostic effects of the factors in Tokuhashi Score, but we did not assess the accuracy of predicted survival time for patients with various levels of Tokuhashi scores.

\section{Conclusion}

Factors included in RTS were all significant on prognostic predicting for patients with spinal metastasis and should be considered when choosing the appropriate treatment modality. What's more, we believe that more accurate prognosis may be obtained by merging patients with KPS 10-40/KPS 50-70 and patients with single/ double involved vertebrae together. Using the modified RTS, patients present with a low score are predicted to live a short period and some palliative therapies should be applied, while patients should be treated with invasive procedures when present with a high RTS score. Additionally, we suggest that more sufficiently considering on the effect of modern therapies is necessary for developing new scores in the future, as adjuvant interventions have significantly altered the patients' life expectancy in the recent years.

\section{Additional file}

Additional file 1: Appendix 1. Searching strategies used for the literature retrieving. (DOCX $13 \mathrm{~kb}$ )

\section{Abbreviations}

Cl: confidence interval; ECOG: Eastern Cooperative Oncology Group; HR: hazard ratio; MSCC: metastatic spinal cord compression; NOS: the Newcastle-Ottawa Scale; PS: performance status; RTS: revised Tokuhashi Score

\section{Acknowledgments}

Not applicable.

\section{Funding}

This research did not receive any specific grant from funding agencies in the public, commercial, or not-for-profit sectors.

\section{Availability of data and materials}

The authors declare that all the data supporting the findings of this study are available within the article and its supplementary information files.

\section{Authors' contributions}

YXG: methodology, validation, formal analysis, investigation, data curation, writingoriginal draft, writing-reviewing and editing, project administration. LDX: investigation, writing-reviewing and editing. HYC: conceptualization, methodology, validation, investigation, writing-reviewing and editing. LYH: methodology, validation, investigation, writing-reviewing and editing. WF: conceptualization, methodology and validation. FJT: formal analysis, investigation and data curation. HKC: writing original draft. YL: writing-reviewing and editing. $\mathrm{ZH}$ : validation and investigation. XMY: project administration, supervision. ZHR: investigation and data curation. All authors read and approved the final manuscript.

\section{Ethics approval and consent to participate}

Not applicable.

\section{Consent for publication}

Not applicable.

\section{Competing interests}

The authors declare that they have no competing interests.

\section{Publisher's Note}

Springer Nature remains neutral with regard to jurisdictional claims in published maps and institutional affiliations.

\section{Author details}

${ }^{1}$ Department of Bone Tumor of Tianjin Hospital, Tianjin 300211, China. ${ }^{2}$ Weifang People's Hospital, Shandong 261000, China. ${ }^{3}$ Tianjin Medical university, Tianjin 300070, China.

Received: 18 April 2018 Accepted: 27 November 2018

Published online: 13 December 2018

\section{References}

1. Byrne TN. Spinal cord compression from epidural metastases. N Engl J Med. 1992;327:614-9.

2. Jacobs WB, Perrin RG. Evaluation and treatment of spinal metastases: an overview. Neurosurg Focus. 2001;11:e10.

3. Tokuhashi Y, Matsuzaki H, Toriyama S, Kawano H, Ohsaka S. Scoring system for the preoperative evaluation of metastatic spine tumor prognosis. Spine. 1990;15:1110-3.

4. Tokuhashi $Y$, Matsuzaki H, Oda H, Oshima M, Ryu J. A revised scoring system for preoperative evaluation of metastatic spine tumor prognosis. Spine. 2005;30:2186-91.

5. Sioutos PJ, Arbit E, Meshulam CF, Galicich JH. Spinal metastases from solid tumors. Analysis of factors affecting survival. Cancer. 1995;76:1453-9.

6. Tomita K, Kawahara N, Kobayashi T, et al. Surgical strategy for spinal metastases. Spine (Phila Pa 1976). 2001;26(3):298-306.

7. Bauer HC, Wedin R. Survival after surgery for spinal and extremity metastases. Prognostication in 241 patients. Acta Orthop Scand. 1995;66:143-6.

8. North RB, LaRocca VR, Schwartz J, et al. Surgical management of spinal metastases: analysis of prognostic factors during a 10-year experience. J Neurosurg: Spine. 2005;2:564-73.

9. Van der Linden YM, Dijkstra SP, Vonk EJ, et al. Prediction of survival in patients with metastases in the spinal column. Results based on a randomized trial of radiotherapy. Cancer. 2005;103:320-8.

10. Quraishi NA, Manoharan SR, Arealis G, et al. Accuracy of the revised Tokuhashi score in predicting survival in patients with metastatic spinal cord compression (MSCC). Eur Spine J. 2013;22(Suppl 1):S21-6.

11. Pointillart V, Vital JM, Salmi R, et al. Survival prognostic factors and clinical outcomes in patients with spinal metastases. J Cancer Res Clin Oncol. 2011; 137(5):849-56.

12. Wells GA, Shea B, O'Connell D et al. Newcastle-Ottawa Quality Assessment scale Cohort Studies. 2012. http://www.ohri.ca/programs/clinical_ epidemiology/oxford.asp.

13. Higgins JP, Thompson SG, Deeks JJ, et al. Measuring inconsistency in metaanalyses. BMJ. 2003;327:557-60.

14. Egger M, Davey Smith G, Schneider M, et al. Bias in meta-analysis detected by a simple, graphical test. BMJ. 1997;315:629-34.

15. Duval S, Tweedie R. Trim and fill: a simple funnel-plot-based method of testing and adjusting for publication bias in meta-analysis. Biometrics. 2000;56:455-63.

16. Lei $M$, Liu Y, Yan L, et al. A validated preoperative score predicting survival and functional outcome in lung cancer patients operated with posterior decompression and stabilization for metastatic spinal cord compression. Eur Spine J. 2016;25(12):3971-8.

17. Lei M, Liu Y, Liu S, et al. Individual strategy for lung cancer patients with metastatic spinal cord compression. Eur Spine J. 2016;42(5):728-34.

18. Lei $M$, Liu $Y$, Tang $C$, et al. Prediction of survival prognosis after surgery in patients with symptomatic metastatic spinal cord compression from nonsmall cell lung cancer. BMC Cancer. 2015;15:853.

19. Rades D, Huttenlocher S, Evers JN, et al. Do elderly patients benefit from surgery in addition to radiotherapy for treatment of metastatic spinal cord compression. Strahlenther Onkol. 2012;188(5):424-30.

20. Rades D, Huttenlocher S, Bajrovic A, et al. Surgery followed by radiotherapy versus radiotherapy alone for metastatic spinal cord compression from unfavorable tumors. Int J Radiat Oncol Biol Phys. 2011;81(5):e861-8.

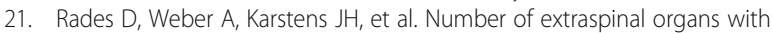
metastases: a prognostic factor of survival in patients with metastatic spinal 
cord compression (MSCC) from non-small cell lung cancer (NSCLC). Anticancer Res. 2014;34(5):2503-7.

22. Rades D, Fehlauer F, Schulte R, et al. Prognostic factors for local control and survival after radiotherapy of metastatic spinal cord compression. J Clin Oncol. 2006;24(21):3388-93.

23. Patchell $R$, Tibbs PA, Regine WF, et al. Direct decompressive surgical resection in the treatment of spinal cord compression caused by metastatic cancer: a randomised trial. Lancet. 2005;366:643-8.

24. Chen YJ, Chang GC, Chen HT, et al. Surgical results of metastatic spinal cord compression secondary to non-small cell lung cancer. Spine (Phila Pa 1976). 2007;32(15):E413-8.

25. Leithner A, Radl R, Gruber G, et al. Predictive value of seven preoperative prognostic scoring systems for spinal metastases. Eur Spine J. 2008;17(11):1488-95.

26. Park JH, Rhim SC, Jeon SR. Efficacy of decompression and fixation for metastatic spinal cord compression: analysis of factors prognostic for survival and postoperative ambulation. J Korean Neurosurg Soc. 2011;50(5):434-40.

27. Arrigo RT, Kalanithi $P$, Cheng I, et al. Predictors of survival after surgical treatment of spinal metastasis. Neurosurgery. 2011;68(3):674-81 discussion 681.

28. Rades D, Douglas S, Veninga T, et al. Metastatic spinal cord compression in non-small cell lung cancer patients. Prognostic factors in a series of 356 patients. Strahlenther Onkol. 2012;188(6):472-6.

29. Crnalic S, Löfvenberg R, Bergh A, et al. Predicting survival for surgery of metastatic spinal cord compression in prostate cancer: a new score. Spine (Phila Pa 1976). 2012;37(26):2168-76.

30. Chong S, Shin SH, Yoo H, et al. Single-stage posterior decompression and stabilization for metastasis of the thoracic spine: prognostic factors for functional outcome and patients' survival. Spine J. 2012;12(12):1083-92.

31. Rades D, Hueppe M, Schild SE. A score to identify patients with metastatic spinal cord compression who may be candidates for best supportive care. Cancer. 2013;119(4):897-903.

32. Ju DG, Zadnik PL, Groves ML, et al. Factors associated with improved outcomes following decompressive surgery for prostate cancer metastatic to the spine. Neurosurgery. 2013;73(4):657-66 discussion 666.

33. Bakker NA, Coppes MH, Vergeer RA, et al. Surgery on spinal epidural metastases (SEM) in renal cell carcinoma: a plea for a new paradigm. Spine J. 2014;14(9):2038-41.

34. Bollen $\mathrm{L}$, van der Linden $\mathrm{YM}$, Pondaag $\mathrm{W}$, et al. Prognostic factors associated with survival in patients with symptomatic spinal bone metastases: a retrospective cohort study of 1,043 patients. Neuro-Oncology. 2014;16(7):991-8.

35. Vanek P, Bradac O, Trebicky F, et al. Influence of the preoperative neurological status on survival after the surgical treatment of symptomatic spinal metastases with spinal cord compression. Spine (Phila Pa 1976). 2015;40(23):1824-30.

36. Tang Y, Qu J, Wu J, et al. Metastatic spinal cord compression from nonsmall-cell lung Cancer treated with surgery and adjuvant therapies: a retrospective analysis of outcomes and prognostic factors in 116 patients. J Bone Joint Surg Am. 2015;97(17):1418-25.

37. Chen YJ, Chen HT, Hsu HC. Preoperative palsy score has no significant association with survival in non-small-cell lung cancer patients with spinal metastases who undergo spinal surgery. J Orthop Surg Res. 2015;10:149.

38. Meng T, Chen R, Zhong N, et al. Factors associated with improved survival following surgical treatment for metastatic prostate cancer in the spine: retrospective analysis of 29 patients in a single center. World J Surg Oncol. 2016;14(1):200.

39. Park SJ, Lee CS, Chung SS. Surgical results of metastatic spinal cord compression (MSCC) from non-small cell lung cancer (NSCLC): analysis of functional outcome, survival time, and complication. Spine J. 2016;16(3):322-8.

40. Huddart RA, Rajan B, Law M, et al. Spinal cord compression in prostate cancer: treatment outcome and prognostic factors. Radiother Oncol. 1997; 44(3):229-36.

41. Williams BJ, Fox BD, Sciubba DM, et al. Surgical management of prostate cancer metastatic to the spine. J Neurosurg Spine. 2009;10(5):414-22.

42. Rades D, Douglas $S$, Veninga T, et al. A survival score for patients with metastatic spinal cord compression from prostate cancer. Strahlenther Onkol. 2012;188(9):802-6.

43. Crnalic S, Hildingsson C, Wikström P, et al. Outcome after surgery for metastatic spinal cord compression in 54 patients with prostate cancer. Acta Orthop. 2012;83(1):80-6.

44. Lee BH, Park JO, Kim HS, et al. Perioperative complication and surgical outcome in patients with spine metastases: retrospective 200-case series in a single institute. Clin Neurol Neurosurg. 2014;122:80-6.
45. Sellin JN, Suki D, Harsh V, et al. Factors affecting survival in 43 consecutive patients after surgery for spinal metastases from thyroid carcinoma. J Neurosurg Spine. 2015;23(4):419-28.

46. Drzymalski DM, Oh WK, Werner $\mathrm{L}$, et al. Predictors of survival in patients with prostate cancer and spinal metastasis. Presented at the 2009 joint spine section meeting. Clinical article. J Neurosurg Spine. 2010;13(6):789-94

47. Tancioni F, Navarria P, Pessina F, et al. Assessment of prognostic factors in patients with metastatic epidural spinal cord compression (MESCC) from solid tumor after surgery plus radiotherapy: a single institution experience. Eur Spine J. 2012;21(Suppl 1):S146-8.

48. Tatsui CE, Suki D, Rao G, et al. Factors affecting survival in 267 consecutive patients undergoing surgery for spinal metastasis from renal cell carcinoma. J Neurosurg Spine. 2014;20(1):108-16.

49. Petteys RJ, Spitz SM, Goodwin CR, et al. Factors associated with improved survival following surgery for renal cell carcinoma spinal metastases. Neurosurg Focus. 2016;41(2):E13.

50. Rades D, Janssen $S$, Käsmann $L$, et al. Outcomes after irradiation of epidural spinal cord compression due to metastatic thyroid Cancer. Anticancer Res. 2016;36(4):2035-9.

51. Kato S, Murakami H, Demura S, et al. The impact of complete surgical resection of spinal metastases on the survival of patients with thyroid cancer. Cancer Med. 2016;5(9):2343-9.

52. Sciubba DM, Gokaslan ZL, Suk I et al. Positive and negative prognostic variables for patients undergoing spine surgery for metastatic breast disease. Eur Spine J 2007 16(10):1659-1667. Epub. 2007 May 8.

53. Walcott BP, Cvetanovich GL, Barnard ZR, et al. Surgical treatment and outcomes of metastatic breast cancer to the spine. J Clin Neurosci. 2011; 18(10):1336-9.

54. Tancioni F, Navarria P, Mancosu P, et al. Surgery followed by radiotherapy for the treatment of metastatic epidural spinal cord compression from breast cancer. Spine (Phila Pa 1976). 2011;36(20):E1352-9.

55. Zadnik PL, Hwang L, Ju DG, et al. Prolonged survival following aggressive treatment for metastatic breast cancer in the spine. Clin Exp Metastasis. 2011;31(1):47-55.

56. Ulmar B, Naumann U, Catalkaya $S$ et al. Prognosis scores of Tokuhashi and Tomita for patients with spinal metastases of renal cancer. Ann Surg Oncol 2006 14(2):998-1004. Epub. 2006 Nov 3.

57. Jiang L, Ouyang H, Liu X, et al. Surgical treatment of 21 patients with spinal metastases of differentiated thyroid cancer. Chin Med J. 2014; 127(23):4092-6.

58. Oliveira MF, Rotta JM, Botelho RV. Survival analysis in patients with metastatic spinal disease: the influence of surgery, histology, clinical and neurologic status. Arq Neuropsiquiatr. 2015;73(4):330-5.

59. Kataoka M, Kunisada T, Tanaka M, et al. Statistical analysis of prognostic factors for survival in patients with spinal metastasis. Acta Med Okayama. 2012:66(3):213-9.

60. Aoude A, Fortin M, Aldebeyan S, et al. The revised Tokuhashi score; analysis of parameters and assessment of its accuracy in determining survival in patient afflicted with spinal metastasis. Eur Spine J. 2018;27(4):835-840.

61. Bartels RH, Feuth $T$, van der Maazen $\mathrm{R}$, et al. Development of a model with which to predict the life expectancy of patients with spinal epidural metastasis. Cancer. 2007;110(9):2042-9.

62. Lei M, Li J, Liu Y, et al. Who are the best candidates for decompressive surgery and spine stabilization in patients with metastatic spinal cord compression? Spine (Phila Pa 1976). 2016;41(18):1469-76.

63. Chang SS, Luo JC, Chao Y, et al. The clinical features and prognostic factors of hepatocellular carcinoma patients with spinal metastasis. Eur 」 Gastroenterol Hepatol. 2001;13(11):1341-5.

64. Chen H, Xiao J, Yang X, et al. Preoperative scoring systems and prognostic factors for patients with spinal metastases from hepatocellular carcinoma. Spine (Phila Pa 1976). 2010;35(23):E1339-46.

65. Choi C, Seong J. Predictive factors of palliative radiotherapy response and survival in patients with spinal metastases from hepatocellular carcinoma. Gut Liver. 2015;9(1):94-102.

66. Guo Y, Young B, Palmer JL, et al. Prognostic factors for survival in metastatic spinal cord compression: a retrospective study in a rehabilitation setting. Am J Phys Med Rehabil. 2003;82(9):665-8.

67. Moon KY, Chung CK, Jahng TA, et al. Postoperative survival and ambulatory outcome in metastatic spinal tumors : prognostic factor analysis. J Korean Neurosurg Soc. 2011;50(3):216-23. 
68. Yang SB, Cho W, Chang UK. Analysis of prognostic factors relating to postoperative survival in spinal metastases. J Korean Neurosurg Soc. 2012; 51(3):127-34.

69. Helweg-Larsen S, Sørensen PS, Kreiner S. Prognostic factors in metastatic spinal cord compression: a prospective study using multivariate analysis of variables influencing survival and gait function in 153 patients. Int J Radiat Oncol Biol Phys. 2000;46(5):1163-9.

70. Kumar N, Tan JJ, Zaw AS, et al. Evaluation of scoring systems and prognostic factors in patients with spinal metastases from nasopharyngeal carcinoma. Spine J. 2014;14(12):2946-53.

71. Mizumoto M, Harada H, Asakura H, et al. Prognostic factors and a scoring system for survival after radiotherapy for metastases to the spinal column: a review of 544 patients at Shizuoka Cancer center hospital. Cancer. 2008; 113(10):2816-22

72. Ogihara S, Seichi A, Hozumi T, et al. Prognostic factors for patients with spinal metastases from lung cancer. Spine (Phila Pa 1976). 2006;31 (14):1585-90.

73. Rades $D$, Veninga $T$, Stalpers $L$, et al. Prognostic factors predicting functional outcomes, recurrence-free survival, and overall survival after radiotherapy for metastatic spinal cord compression in breast cancer patients. Int J Radiat Oncol Biol Phys. 2006;64(1):182-8.

74. Switlyk MD, Kongsgaard U, Skjeldal S, et al. Prognostic factors in patients with symptomatic spinal metastases and normal neurological function. Clin Oncol (R Coll Radiol). 2015;27(4):213-21.

75. Tao HM, Ye ZM, Yang DS, et al. Risk factors and prognosis of surgery for spinal metastasis. Zhonghua Zhong Liu Za Zhi. 2004;26(4):226-30.

76. Weber A, Bartscht T, Karstens JH, et al. Breast cancer patients with metastatic spinal cord compression: Number of extraspinal organs involved by metastases influences survival. Strahlenther Onkol. 2013:1-4.

77. Yamashita T, Siemionow KB, Mroz TE, et al. A prospective analysis of prognostic factors in patients with spinal metastases: use of the revised Tokuhashi score. Spine (Phila Pa 1976). 2011;36(11):910-7.

78. Yeung YN, Cheung KK, Lam TC, et al. A study of the predictive value of the modified Tokuhashi score in metastatic spinal tumour causing cord compression in a southern Chinese population. J Orthop, Trauma Rehabil. 2014:1-7.

79. Zhang D, Xu W, Liu T, et al. Surgery and prognostic factors of patients with epidural spinal cord compression caused by hepatocellular carcinoma metastases: retrospective study of 36 patients in a single center. Spine (Phila Pa 1976). 2013;38(17):E1090-5.

80. Enkaoua EA, Doursounian L, Chatellier G, et al. Vertebral metastases: a critical appreciation of the preoperative prognostic tokuhashi score in a series of 71 cases. Spine (Phila Pa 1976). 1997;22(19):2293-8.

81. Gakhar H, Swamy GN, Bommireddy R, et al. A study investigating the validity of modified Tokuhashi score to decide surgical intervention in patients with metastatic spinal cancer. Eur Spine J. 2013;22(3):565-8.

82. Sandler A, Gray R, Perry MC, et al. Paclitaxel-carboplatin alone or with bevacizumab for non-small-cell lung cancer. N Engl J Med. 2006;355(24): 2542-50.

83. Horn L, Sandler A. Epidermal growth factor receptor inhibitors and antiangiogenic agents for the treatment of non-small cell lung cancer. Clin Cancer Res. 2009;15(16):5040-8.

Ready to submit your research? Choose BMC and benefit from:

- fast, convenient online submission

- thorough peer review by experienced researchers in your field

- rapid publication on acceptance

- support for research data, including large and complex data types

- gold Open Access which fosters wider collaboration and increased citations

- maximum visibility for your research: over $100 \mathrm{M}$ website views per year

At BMC, research is always in progress.

Learn more biomedcentral.com/submissions 